\title{
Introduction to the Special Issue: Teaching AAC Use to Individuals with Developmental and Physical Disabilities
}

\author{
Jeff Sigafoos ${ }^{1}$
}

Published online: 19 December 2015

(C) Springer Science+Business Media New York 2015

Individuals with developmental and physical disabilities also often have severe communication impairment or complex communication needs (Beukelman and Mirenda 2013). The term severe communication impairment refers to situations where the person's speech is so limited or unintelligible that it is unable to meet his or her everyday communication needs. Lack of sufficient, intelligible speech is prevalent among individuals with developmental and physical disabilities. McQueen et al. (1987), for example, found that approximately $65 \%$ of children with significant intellectual disability had limited or no intelligible speech. Among children with autism spectrum disorder, Tager-Flusberg and Kasari (2013) noted that about $30 \%$ are likely to "remain with little expressive spoken language ability by the time they reach school age." (p. 468).

The high prevalence of severe communication impairment among individuals with developmental and physical disabilities makes communication intervention a major habilitation priority for this population. AAC intervention requires identifying suitable non-speech communication modalities, as well as teaching the person to use non-speech modes for effective communication. To this end, AAC research is often focused on developing new and more effective intervention approaches.

This special issue of the Journal of Developmental and Physical Disabilities was commissioned to attract papers that focused on developing new and more effective intervention approaches for individuals with developmental and physical disabilities and severe communication impairment. The goal of this special issue is to provide a forum for researchers using a broad range of AAC systems and intervention approaches to share the results of their recent efforts, be they empirical or conceptual. A secondary

Jeff Sigafoos

jeff.sigafoos@vuw.ac.nz

1 School of Education, Victoria University of Wellington, PO Box 17-310, Karori, 6147 Wellington, New Zealand 
goal was to identify directions for future research so as to advance the use of AAC among individuals with developmental and physical disabilities. The nine papers included in this special issue cover a range of topics related to the provision of AAC intervention to individuals with developmental and physical disabilities.

The paper by Tönsing, for example, focused on teaching symbol combinations to four children with developmental disabilities. The children received instruction with two types of AAC devices; an Apple iPad ${ }^{\circledR}$ with speech-generating software and a low-tech communication board. Their aim was to compare learning of symbol combinations with the two devices. An interesting twist was that the children and their communication partners were also assessed to determine which of the two devices they preferred. Through this study, Tönsing not only contributes new procedures for teaching symbol combinations, but she also demonstrates the application of a rigorous research methodology for comparing different types of AAC devices in terms of learning performance and preference. Comparative research of this type is extremely helpful with respect to identifying the relative merits of different AAC devices. Data of this type can, in turn, be quite useful to practitioners who are faced with the challenging task of identifying suitable AAC modes that would enable children to progress from producing single symbol communication responses to producing multi-symbol combinations.

In the second paper, Rose, Trembath, and Bloomberg studied the relation between children's degree of visual attending and performance during an intervention in which key word signs (i.e., the use of a manual sign simultaneously with speech) were modeled. Their findings highlight the complexity of identifying relations between looking (attending) and doing (signing). Their paper will no doubt stimulate future research into the impact of attending on the acquisition of AAC skills.

The paper by Simacek, Reichle, and McComas represents a classic intervention study with its aim to evaluate the effectiveness of a set of instructional procedures for teaching two individuals with Rett syndrome to communicate using a speechgenerating device. The results demonstrated effective procedures for teaching the participants to make requests with a high-tech AAC device. This is an important demonstration because the prevailing wisdom was that this type of educational/ behavioral intervention was largely ineffective for children with Rett syndrome (Smith et al. 1995). Perhaps the key to the success in this study was that the researchers' successfully identified ways for the children to access their AAC device that were compatible with the participant's existing abilities.

In the fourth paper, Lancioni and his colleagues evaluated a novel speech-generating device for participants with multiple disabilities that included vision, motor, and hearing impairments. The technology the used for enabling the participants to operate the speech-generating devices is extremely innovative and shows creative problem solving at its best. The side of the device facing the participants was divided into 15 sections each containing an optic sensor. Above each optic sensor, there was a small object or tag with a word in Braille referring to an activity. The device was set-up so that removing an object/tag triggered the underlying optic sensor and caused the device to verbalize a request. This device allowed each participant to independently choose among different activities and establish successful communication with the caregiver. Perhaps the most important aspect of this new technology is that it proved to be a highly effective way of enabling the caregiver to play an effective mediation role. 
The next paper by Townend et al. makes use of advances in eye gaze technology to open the door to communication for individuals who might otherwise be unable to access high-tech AAC systems due to the severity of their physical impairment. Making best use of technology, however, is about more than the potential of the device. Taking the experiences of Dutch families of individuals with Rett syndrome as an exemplar, their paper suggests that the value of the input from suppliers and knowledgeable AAC professionals should not be underestimated. They argue that training and support for individuals, families, and other communication partners should not be regarded as an add-on or optional extra, but should be seen as an integral part of the package when any eye gaze system is provided.

Following this, Chung, Snodgrass, Meadan, Akamoglu, and Halle offer a unique exploration into the potential of a mixed-methods approach in AAC research; an approach that values equally the quantitative and qualitative methods (i.e., observational behavioral recording and self-report social validity assessment) for evaluating single-case research. Their paper addresses the challenges faced when these two methods produce discrepant findings. They examine one potential explanation for discrepant findings by conducting a secondary data analysis. It is important to note the eventual goal of their work, which is the enhancement of the children's AAC use via parent-implemented milieu (i.e., naturalistic) strategies. Perhaps, most relevant for future research is the fact that their secondary analysis was more granular, which revealed changes in children's AAC topography of responding that the original (and more generic) recording of initiations and responses concealed. Their paper thus offers an important lesson for undertaking data analysis in mixed-methods studies.

In the seventh paper, Ogletree et al. report on an intervention study focused on training three communication partners of an adult AAC user. Their data show that the training model was effective in supporting partners in the use of more effective communication strategies. The authors argue convincingly for giving increased consideration to partner training as a vehicle to promote broad-based AAC success. This is a critically important study because most AAC intervention research is focused solely on teaching the AAC user to use AAC. But, as Ogletree et al. argue, it is also critically important to ensure that communication partners know how to effectively interact with and support AAC users.

The penultimate paper by Hudson, Zambone, and Brickhouse focuses on teaching early numeracy skills using AAC. This group of special education researchers build a strong case for the importance of teaching early numeracy skills. For one, such skills are important for understanding higher-level mathematics. They argue that all students, including students with severe multiple disabilities, need access to appropriate gradelevel academic instruction. Despite this, it is clear that many special educators struggle with the challenging task of teaching early numeracy skills, due in part to the difficulty that some students experience in responding to and accessing the curriculum. For example, how do nonverbal students count by rote to 5? How do students with blindness add sets to answer a question, such as: How many altogether? Examples or models for teaching early numeracy (or any academic content) to such students are virtually nonexistent. To move the literature forward in this area, their study evaluated the effects of an early numeracy curriculum on early numeracy skill acquisition for students who required AAC. Their results demonstrate an effective model for enabling access to the curriculum via AAC. 
The final paper by Schlosser et al. offers a conceptualization of the just-in-time (JIT) construct. They provide a strong rationale for viewing JIT as a promising and innovative approach to the delivery of AAC services and interventions. Indeed, JIT would seem to have profound implications for vocabulary selection, vocabulary organizations, and vocabulary instructions. Readers of this paper will come away with an understanding of the origins of this construct in the business world, the history of its application in the AAC field, and an overview of the theoretical underpinnings of the potential benefits of JIT. The authors provide a valuable service in also offering a proposed taxonomy that illustrates the diverse ways in which clinicians and educators could adopt a JIT approach to advance their implementation of AAC. Their scholarly discussion of the role of communication partners and learners alike provides a blue print of sorts as to the new demands and opportunities JIT affords.

Together, the nine papers in this special issue cover a range of topics and research approaches that reflect the incredibly rich diversity of contemporary AAC research and practice involving individuals with developmental and physical disabilities. These efforts will propel AAC research forward in new and exciting directions and will surely open the door to communication for many more people who might otherwise remain unable to communicate. The collective results of these studies and the conceptual papers suggest that effective AAC intervention requires creative approaches to designing the technology and access options so as to enable persons with developmental and physical disabilities to use AAC. More specifically, these papers have highlighted the need for careful implementation of systematic instructional procedures for teaching AAC use, the need to consider the person's existing abilities, the need to support communication partners, and the value of considering new methods of conceptualizing AAC interventions for individuals with developmental and physical disabilities. I am indebited to the authors for their excellent contribtions to this special issue and for providing me with a summary of their articles that I have used in this editorial introduction.

Conflict of Interest The author reports no conflict of interests.

\section{References}

Beukelman, D., \& Mirenda, P. (2013). Augmentative and alternative communication: Supporting children and adults with complex communication needs (4th ed.). Baltimore: Paul H. Brookes Publishing Co.

McQueen, P. C., Spence, M. W., Garner, J. B., Pereira, L. H., \& Winsor, E. J. T. (1987). Prevalence of major mental retardation and associated disabilities in the Canadian maritime provinces. American Journal of Mental Deficiency, 91, 460-466.

Smith, T., Klevstrand, M., \& Lovaas, O. I. (1995). Behavioral treatment of Rett's disorder: ineffectiveness in three cases. American Journal on Mental Retardation, 100, 317-322.

Tager-Flusberg, H., \& Kasari, C. (2013). Minimally verbal school-aged children with autism spectrum disorder: the neglected end of the spectrum. Autism Research, 6, 468-478. doi:10.1002/aur.1329. 